\title{
Using Game Theory Approach for Assessment of Risk and Police Patrols Scheduling
}

\author{
Cheng-Kuang Wu, Chih-Chun Hou \\ School of Computer Science and Software Engineering \\ Zhaoqing University
}

Zhaoqing City, Guangdong Province, China

shapleyvalue@hotmail.com

\section{基于博奕分析风险模型建置公安巡逻配置班表}

\author{
吴正光，侯志君 \\ 肇庆学院计算机科学与软件学院、大数据学院 \\ 广东省肇庆市 526021，中国 \\ shapleyvalue@hotmail.com
}

\begin{abstract}
This study proposes a two-step game theory model framework for the purpose of scheduling police patrol shifts. In the first step, the three important factors of interaction between criminals and police are modeled as a zero-sum noncooperative game, and the risk value of each patrol area is obtained by calculating the mixed strategy Nash equilibrium. In the second step, the Shapley values of three different threat levels are calculated according to the rates of criminal events within three time periods. Finally, the Shapley value is derived based on the three criminal threat levels for each district. A fair allocation of police personnel based on the Shapley value is made with a minimum set of personnel deployment costs. This study used the 2016 San Francisco Public Security Bureau's open data for consolidation and calculations to validate the model. The experimental results show that police planners can use this framework to quantitatively evaluate the criminal threat in each district when deciding upon the deployment of patrol officers for three shifts per day.
\end{abstract}

Keywords-Nash equilibrium; Risk value; Shapley value; Patrol deployment

摘要一本方案结合了两种博弯理论模型, 进而计算出各区 域每日三班的公安巡逻服务时间配置表。第 1 模型将各区域的 犯罪者与公安领导之间的互动关系建构成一场非合作零合博 奕, 计算博奕的混合随机策略纳许均衡解, 当作区域的安全 风险值。第 2 模型根据公安局记录所有发生案件的次数, 计算 三个时段发生案件次数的比例, 当作三个安全警示等级的三 个门坎值, 依三个不同安全警示等级的门坎值, 计算所有区 域安全威胁值的结盟数值, 并得出每一区域的夏普利值

（Shapley value）。最后, 将各区域的夏普利值乘上每日三班 次的公安巡逻人员总数, 即产出 10 区域每日三班次的公安巡 逻班表。本研究利用 2016 年旧金山市公安局的开放数据验证 本模型。实验结果显示, 在现有的公安巡逻警力下, 可以有 效的分配整体三时段的巡逻班表。公安部门管理者应用本架 构可以量化评估那个区域是较危险? 以增加公安部门处理犯 罪发生时的反应能力。
关键词一纳许均衡, 风险值, 夏普利值, 巡逻配置

\section{I. 引言}

公安资源有限的城市里，公安局（Police Department） 能在任何时间提供完善充分的安全覆盖是一个极具挑战 性的任务。愈来愈多随机性的犯罪活动, 使得公安编组 巡逻的安排更加困难, 唯有建立优化的公安巡逻时间表, 才能减少潜在的犯罪行为发生。在图 1 中, 公安巡逻存 在着资源分配的困境, 当部署公安越多, 巡逻愈频繁, 巡逻人力及装备耗用的成本就越高, 大规模部署往往会 浪费资源，反而会让都市安全威胁（风险）提升；然而， 部署公安太少, 巡逻次数太少, 或遗漏了重点地区, 只 会让犯罪者有机可乘, 可能引发更多的犯罪事件, 公安 局对于「提升破案能力」和「降低公安巡逻成本」之间 的困境无计可施 ${ }^{[1]}$ 。因此, 完备而适宜的公安巡逻计划, 必须找出公安巡逻数量和破案能力之间的平衡点, 即以 最适宜的数量达到最大的破案能力。现行的巡逻排班缺 乏理性决策的具体措施, 并没有应用数学模型来建构

$\lceil$ 公安警力」和「辖区犯罪者」之间的互动行为。公安 局应该建构一套数据分析工具, 它可以衡量每一区域的 犯罪活动强度 (即犯罪率) 和公安的破案能力 (即破案 率），并需要考虑公安和犯罪者的行动效用（payoff）, 以随机的巡逻策略, 避免犯罪者能够预先观察公安巡逻 表中的漏洞。 


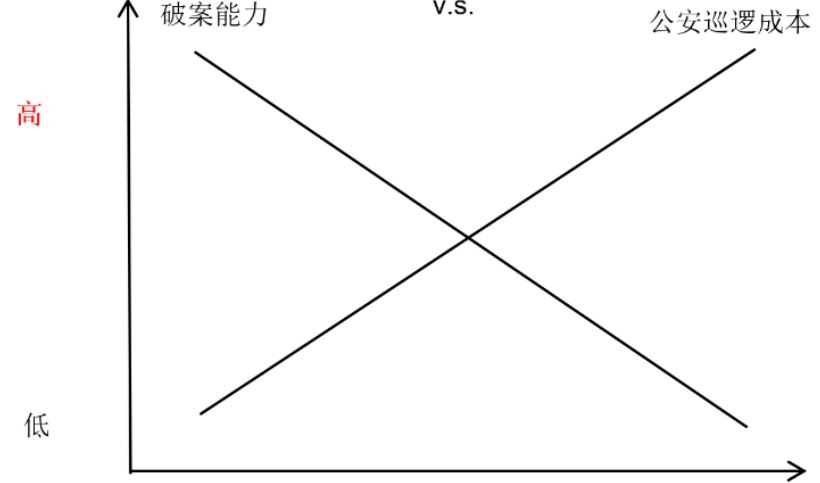

低 安全威胁

次的公安巡逻班表。本研究利用 2016 年旧金山市公安局 的开放数据 [3] 验证本模型。

\section{II. 复合式赛局模型}

本研究专门为安全警示等级系统设计一都市区域公 安巡逻配置的博弯方案, 由两个阶段来计算不同安全等 级(三个时段)的全区域公安巡逻表，图 2 显示，本研究提 供不同安全警示等级, 也就是三个时段优化公安巡逻配 置的流程, 第一阶段计算各区域安全威胁值(TVC), 第 二阶段计算所有区域早、中、晚三时段的公安巡逻配置 表。

\section{第一阶段步骤如下:}

1. 我们将各区域的「犯罪者」及「公安领导」当 作博弯的玩家, 两个玩家的互动过程对应在一

图 1. 降低公安巡逻成本与提升破案能力的困境

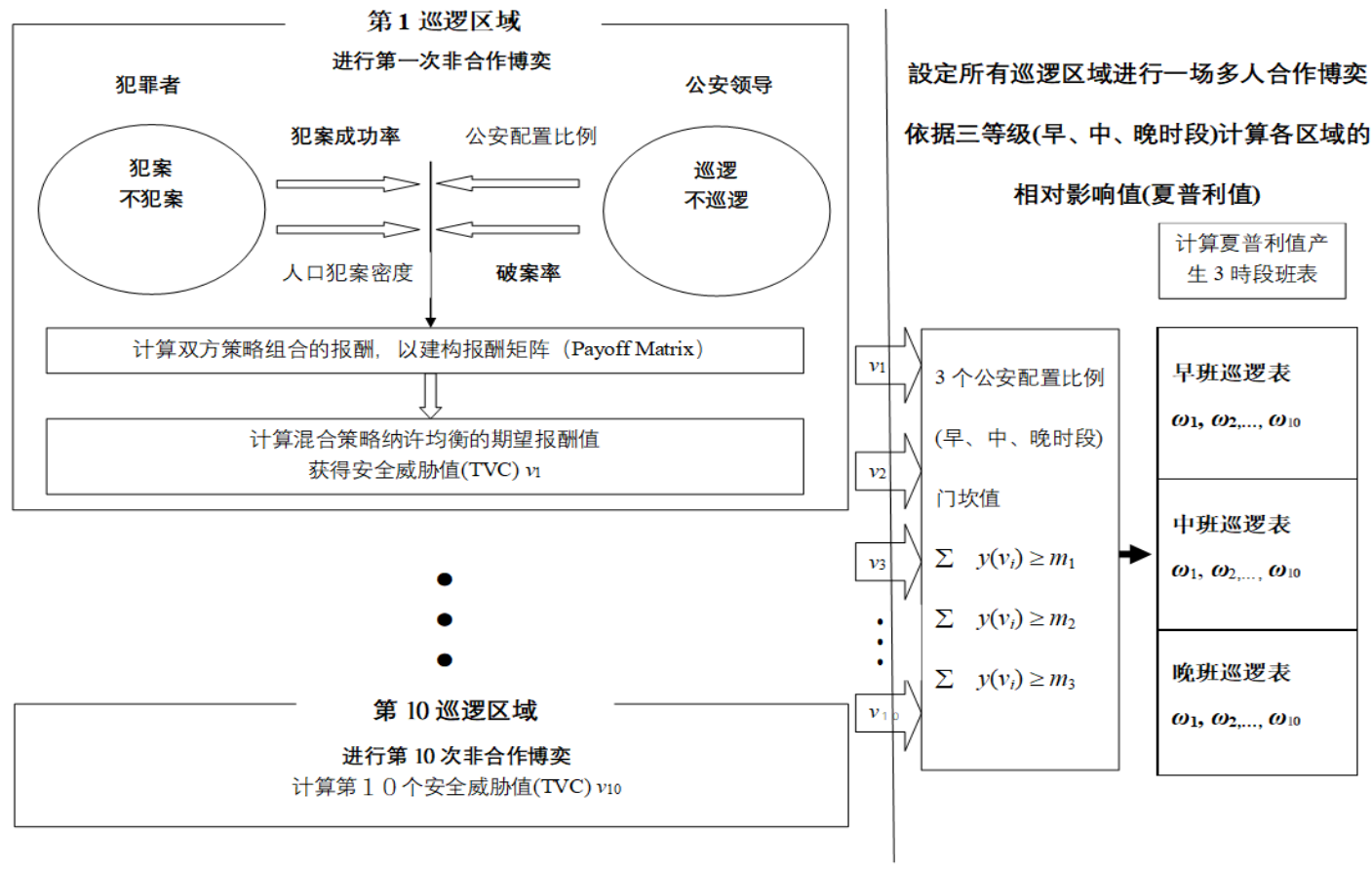

第一阶段

第二阶段

圖 2. 建构 10 区域每日三班次的公安巡逻班表流程图

本研究结合了两种博弯理论模型, 进而计算出各区 域每日三班的公安巡逻服务时间配置表。第 1 模型将各 区域的「犯罪者」与「公安领导」之间的互动关系建构 成一场非合作零合博奕, 计算每个区域的犯案成功率、 公安配置比例和人口犯罪密度, 进而求得博奕的混合随 机策略纳许均衡解, 当作区域的安全风险值（TVC: Threat-Vulnerability-Consequence）。第 2 模型根据公安 局记录所有发生案件的次数(共 149896 笔), 计算三个时 段(早：08:00 15:59; 中: 16:00 23:59; 晚: 00:00 07:59) 发生案件次数的比例, 当作三个安全警示等级的三个门 坎值，依三个不同安全警示等级的门坎值，计算所有区 域安全威胁值的结盟数值, 并得出每一区域的夏普利值

（Shapley value） [2]。最后, 将各区域的夏普利值乘上每 日三班次的公安巡逻人员总数, 即产出 10 区域每日三班
个非合作有限的零和博弯。

2. 考虑测量与评估威胁的影响因素: 人口犯案密 度、犯案成功率、公安配置比例等三个因素。

3. 然后, 计算双方策略组合的报酬, 建构报酬矩 阵, 由这报酬矩阵获得混合随机策略纳许均衡 的期望报酬值, 以这值作为该区域的安全威胁 值(TVC) $v_{i}$ 。

\section{第二阶段步骤如下:}

1. 在 10 个区域的公安领导间建构一个公安警力分 配的合作博弯。

2. 计算各区域早、中、晚班三时段的案件发生次 数, 以早、中、晚班三时段案件的比例当作三 个门坎值, 以划分三个安全警示等级。

3. 依三个不同的安全警示等级的门坎值, 计算所 有区域的相对影响值(夏普利值), 如果安全威胁 
值大于或等于门坎值时, 就累计该区域安全威 胁值。

4. 最后, 得到三个不同安全警示等级的夏普利值 配置集合, 再乘上三时段的公安警力, 得到一 个配置所有区域的早、中、晚三时段的公安巡 逻班表。

\section{A. 非合作安全威胁博奕}

在 10 区域中每 1 区域建构一场安全威胁博亦: 将各 区域的「犯罪者」及「公安领导」双方建构一场非合作 零和安全威胁博竕, 应用 EXCEL 软件计算两个玩家互动 过程的三个影响因子：人口犯罪密度、犯案成功率及公 安配置比例。计算公式如下:

1) 人犯罪密度 $\delta_{i}$ : 计算一年中每日 24 小时, 每小 时的各区域（共 10 区域）犯案数量。计算 2010 年各区域人口数量（只能找到该年的总人口数: 802017 人）。依据前二项数据算出每个区域的 人口犯罪密度。

$$
\text { 第 } i \text { 區域人口犯罪密度 } \delta_{i}=\frac{\text { 第 } i \text { 區域犯案數量 }}{\text { 第 } i \text { 區域人口數量 }}
$$

2) 犯案成功率 $1-\alpha_{i}$ : 计算每时段（早、中、晚） 的犯案数量及破案数量, 并计算出各区域的破 案率及犯案成功率。

$$
\text { 第 } i \text { 區域破案率 } \alpha_{i}=\frac{\text { 第塸域破案數量 }}{\text { 第 } i \text { 品域犯案數量 }}
$$

第 $i$ 區域犯案成功率 $=1-$ 第 $i$ 區域破案率 $=\left(1-\alpha_{i}\right)$

3）公安配置比例 $C_{i}$ : 计算各区域人口比例。

$$
\text { 第 } i \text { 區域人口比例 }=\frac{\text { 第 } i \text { 區域人口總數量 }}{\text { 所有 }(\text { 全 }) \text { 區域人口數量 }}
$$

计算现行各区域公安局公安配置数量, 因为找不到 数据就以人口及全区域公安总数量（2016年为 2292 人）的比例。

第 $i$ 區域现行公安配置數量 $=$ 第 $i$ 區域人口比例 $\times$ 所有（全）區域公安總數量

现行各区域公安配置比例是依人口数量计算:

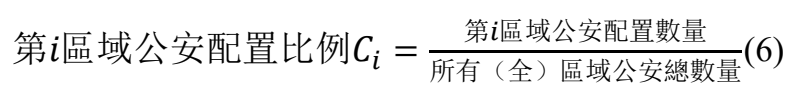

4) 建构两个玩家的互动影响因子的报酬矩阵 (Payoff Matrix)：依据各区域「犯罪者」及「公 安领导」互动过程建构策略组合的报酬矩阵, 以 Bayview 区域为例, 如表 1:
表 1. BAYVIEW 区安全威胁报酬矩阵

\begin{tabular}{|c|c|c|c|}
\hline 报酬矩阵 & \multicolumn{3}{|c|}{ 公安领导 } \\
\hline 犯罪者 & 巡逻 $q$ & 不巡逻 1-q & $q$-mix \\
\hline 犯罪 $p$ & $\left(1-\alpha_{i}\right) \delta_{i} C_{i}$ & $\delta_{i}$ & $\begin{array}{c}\left(1-\alpha_{i}\right) \delta_{i} C_{i} q \\
+\delta_{i}(1-q)\end{array}$ \\
\hline 不犯罪 1- $p$ & $C_{i}$ & 0 & $C_{i} q$ \\
\hline$p$-mix & $\begin{array}{l}\left(1-\alpha_{i}\right) \delta_{i} C_{i} p \\
+C_{i}(1-p)\end{array}$ & $\delta_{i} p$ & $\begin{array}{c}\text { 安全威胁值 } \\
\text { T V C }\end{array}$ \\
\hline
\end{tabular}

本研究的安全威胁博亦没有纯粹策略均衡, 所以用 纯粹策略的随机分布概念, 来计算玩家的混合策略均衡 概率值。犯罪者有「犯罪」与「不犯罪」的两个策略, $p$ 代表「犯罪」的混合策略概率值; 而 1-p 代表「不犯罪」 的混合策略概率值。公安领导有「巡逻」与「不巡逻」 的两个策略, $q$ 代表「巡逻」的混合策略概率值; 而 1-q 代表「不巡逻」的混合策略概率值。经由随机混合策略 纳许均衡算法, 计算出混合策略均衡概率值 $p$ 及 $q$, 这一 概率组合为双方最适策略反应的概率值 ${ }^{[4][5]}$ 。

$$
\begin{gathered}
p=\frac{C_{i}}{\delta_{i}+C_{i}-\left(1-\alpha_{i}\right) \delta_{i} C_{i}} \\
q=\frac{\delta_{i}}{\delta_{i}+C_{i}-\left(1-\alpha_{i}\right) \delta_{i} C_{i}}
\end{gathered}
$$

因为本研究是双人零和博亦, 犯罪者的期望报酬为 正值, 是因为犯罪者从公安领导的缺失得到利益; 而公 安领导的期望报酬为负值, 是因为公安领导预防与处理 犯罪者的案件中付出成本。所以当犯罪者的期望报酬愈 大（值愈大）, 代表犯罪者的威胁愈大, 本研究定义发 生在第 $i$ 区域的安全威胁值 $v_{i}$ 为犯罪者的期望报酬值, 计 算如下:

$v_{i}=p q\left(1-\alpha_{i}\right) \delta_{i} C_{i}+p(1-q) \delta_{i}+(1-p) q C_{i}$

代入方程式(7)(8)得

$$
v_{i}=\frac{\delta_{i}^{2} C_{i}-\delta_{i}{ }^{2} C_{i}^{2}-\alpha_{i} \delta_{i}{ }^{2} C_{i}^{2}}{\left(\delta_{i}+C_{i}-\left(1-\alpha_{i}\right) \delta_{i} C_{i}\right)^{2}}
$$

\section{B. 公安警力分配的合作博亦}

本研究认定在都市中所有发生公安事件的安全威胁 值会互相影响, 以及所有区域的公安领导会建立一个资 源共享与互助的情境, 这情境设定为一个合作博弯。公 安部门需要一个公安警力合理分配的方法, 让管理者可 以依据三个不同时段即三个安全等级去配置 10 个区域的 公安巡逻警力。夏普利值是解合作博竕的一种方法, 近

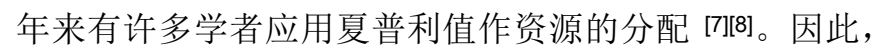
本研究依据三个不同时段的安全警示等级（即早、中、 晚班）计算各区域的夏普利值来分配公安巡逻警力。以 下利用 EXCEL 软件计算早、中、晚班三个时段的门坎值, 并计算各区域三个时段的夏普利值。 
以 2016年旧金山公安局记录所有发生案件的次数(共 149896 笔), 区分三个时段 (早：08:00 15:59; 中: 16:00 23:59; 晚: 00:00 07:59)发生案件的次数的比例, 当作三个安全警示等级的三个门坎值, 如表 2 。接着利 用 EXCEL 按发生犯案的比例和各区域的安全威胁总数来 计算三个时段的门坎值, 如表 2 。

表 2. 安全警示等级的三个门坎值

\begin{tabular}{|l|l|l|l|l|}
\hline $\begin{array}{l}\text { 等 } \\
\text { 级 }\end{array}$ & $\begin{array}{l}\text { 时间范 } \\
\text { 围 }\end{array}$ & $\begin{array}{l}\text { 案件发生次数 } \\
\text { (共 149896案 件 发 }\end{array}$ & $\begin{array}{l}\text { 门坎值 } \\
\text { 生比例 }\end{array}$ \\
\hline 早 & $00: 00 \sim$ & 27187 & $r_{1}=0.181$ & $m_{1}=0.1048$ \\
& $07: 59$ & & & \\
\hline 中 & $08: 00 \sim$ & 56674 & $r_{2}=0.378$ & $m_{2}=0.2184$ \\
& $15: 59$ & & $r_{3}=0.440$ & $m_{3}=0.2544$ \\
\hline 晚 & $16: 00 \sim$ & 66014 & & \\
& $23: 59$ & & & \\
\hline
\end{tabular}

当所有区域的安全威胁值依照表 2 把安全等级分成 3 个等级后, 将这些区域的安全威胁值, 模式化成一个 10 人的合作博亦, 可以依据不同的安全等级呈现出不同的 威胁型态(threat pattern)。我们设定特征函数 [6]: 结盟 $C$ 的群聚值是所有小区域 $\operatorname{TVC}\left(v_{i}\right)$ 的加总 $\sum_{i} \subset_{C} y\left(v_{i}\right)$, 每一个 小区域的威胁值属于其中一个安全等级中的门坎值, 我 们就可以根据不同的安全等级, 计算出各区域的夏普利 值。由于不同的威胁安全等级, 可以反应出每个小区域 对于其它小区域的相对重要性, 设定 $y(C)=\sum_{i} \subset_{C} y\left(v_{i}\right)$, $v_{i} \in V, C \subset X, c$ 是结盟 $C$ 的基数。因此, 可以用方程式 (11)计算第 $t^{t h}$ 个小区域的夏普利值, 计算如下:

$$
\begin{gathered}
\omega(i)=\sum_{\substack{c \subset X \\
i \in c}}^{n} \frac{(c-1) !(n-c) !}{n !}[y(C)-y(C-\{i\})] \\
\Rightarrow \omega(i)=\sum_{\substack{c \subset X \\
i \in c}}^{n} \frac{(c-1) !(n-c) !}{n !}
\end{gathered}
$$

根据表 2 内早、中、晚三时段各区域的夏普利值及 全区域公安配置数量 (总数 2292), 计算各区域三时段 的公安配置数量, 由早、中、晚三时段发生案件的次数 (总数) 的比例 (如表 2) 乘上公安配置总数量 (总数 2292 人), 分别计算如下:

早班公安配置数量 $=0.1814 \times 2292=416$

中班公安配置数量 $=0.3781 \times 2292=867$

晚班公安配置数量 $=0.4405 \times 2292=1010$

再将早、中、晚班配置数量各自乘上各区域的夏普利值, 即得到每日三时段的公安巡逻配置班表。

\section{III. 实验结果}

本研究使用美国旧金山市开放数据库内的 2016 年旧 金山市公安局案件处理登记数据（共 149896 笔）进行挖 掘及计算, 并产出 10 区域的每日三班次的公安巡逻配置 班表。我们分析 10 个区域的四个影响因子, 即人口犯案 密度、人口数量、犯案成功率及公安配置比例, 和本研 究计算出安全威胁值的互相影响关系, 制成图 3、图 4、 5 , 以便于验证本研究计算出安全威胁值的可信度。
犯案成功率与安全威胁值 (TVC) 的关系: 在图 3 中, 当我们固定每个区域的人口犯案密度、人口数量、 犯案数量及公安配置比例, 把这些设为固定值, 然后从 第 1 区到第 10 区逐渐增加公安的破案数量, 也就是犯案 成功率逐渐降低, 发现安全威胁值会逐渐下降, 如图 3, 它代表公安破案的能力逐渐增强, 而犯罪者的期望报酬 也会逐渐减少, 区域的安全威胁值也会随之降低。

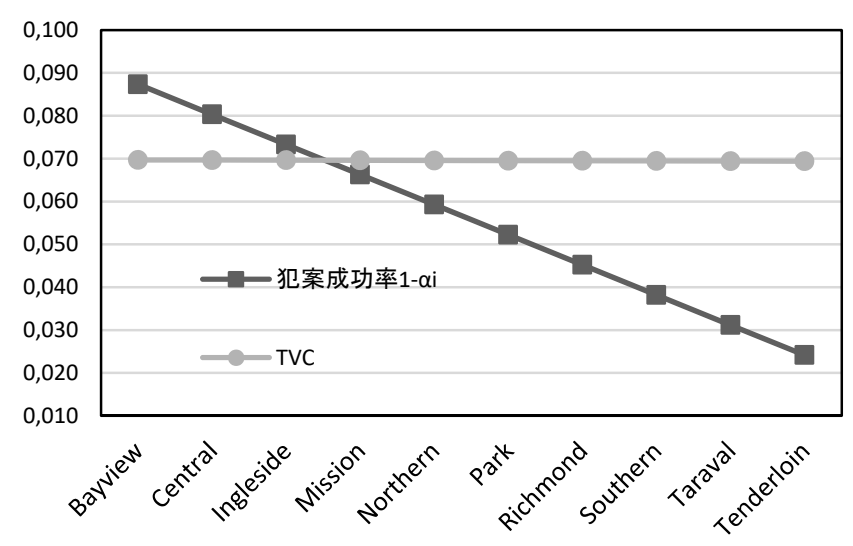

图 3. 犯案成功率与安全威胁值 (TVC) 的关系

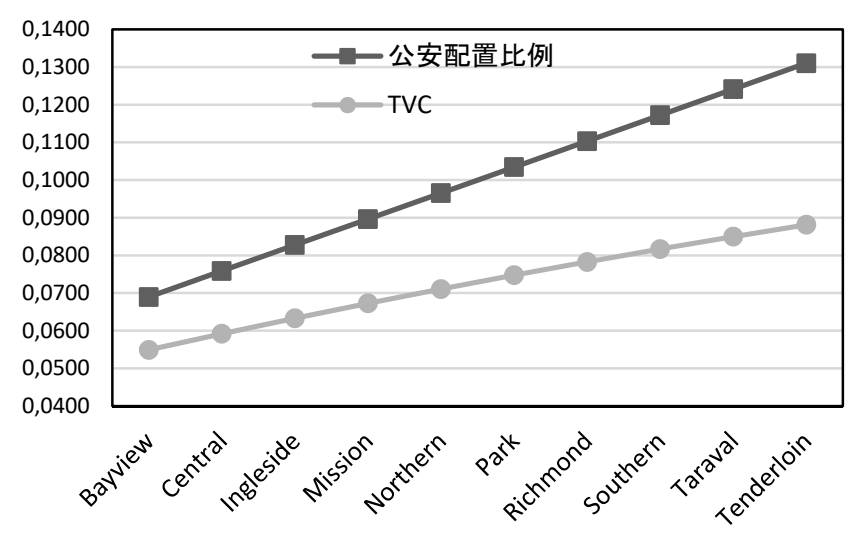

图 4. 公安配置比例与安全威胁值 (TVC) 的关系

公安配置比例与安全威胁值 (TVC) 的关系: 在图 4 中, 当我们固定每个区域的人口数量、犯案数量、公安 的破案率及犯案成功率, 把这些设为固定值, 然后从第 1 区到第 10 区逐渐增加公安的配置数量, 也就是公安配 置比例逐渐上升时, 安全威胁值也会逐渐上升, 如图 4, 它代表公安配置数量愈多, 公安付出的期望报酬愈多, 相对地, 犯罪者得到的期望报酬也会逐渐增加, 区域的 安全威胁值也随之提高, 因此, 公安配置过多, 不只会 造成资源浪费, 也会影响公安破案的能力, 所以公安配 置数量要适宜够用就好。 


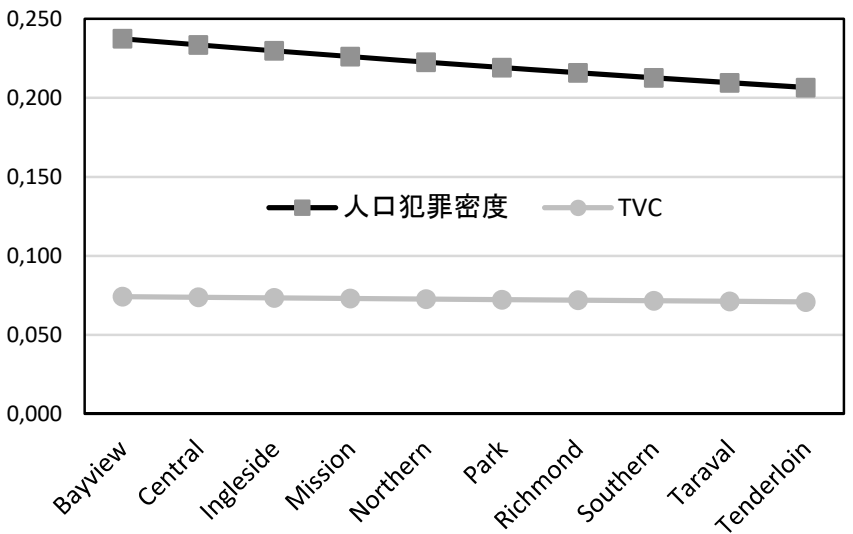

图 5. 人口犯罪密度与安全威胁值（TVC）的关系

人口犯罪密度与安全威胁值 (TVC) 的关系: 在图 5 中, 当我们固定每个区域的公安配置比例、犯案数量、 公安的破案率及犯案成功率, 把这些设为固定值, 然后 从第 1 区到第 10 区逐渐增加人口数量, 由于犯案数量不 变, 采固定值, 而逐渐增加人口数量, 会使得人口犯罪 密度随之降低, 如图 5, 我们发现安全威胁值会逐渐下 降, 它代表人口犯罪密度愈小, 而犯罪者的得到的期望 报酬也会逐渐减少, 区域的安全威胁值就会随之减少, 如图 5。

由以上三点, 我们可以知道区域内的犯案成功率、 公安配置比例及人口犯案密度三个重要因子都和安全威 胁值的关系成正比，也就是说，区域的安全威胁值会随 着三个因子的数值增加而增加, 并随着三个因子的数值 减少而减少。因此, 得知本研究得出的区域安全威胁值 是符合常理的计算方法。从图 6 到图 9, 为三个因子的数 值与安全威胁数值的比较图, 由图 9 中我们可以看到三 个因子 $\alpha_{i}, \delta_{i}, C_{i}$ 由方程式(8) 计算得到安全威胁值 $v_{i}$, 各区 域的三个因子和安全威胁值 (TVC) 是互相关联及影响。

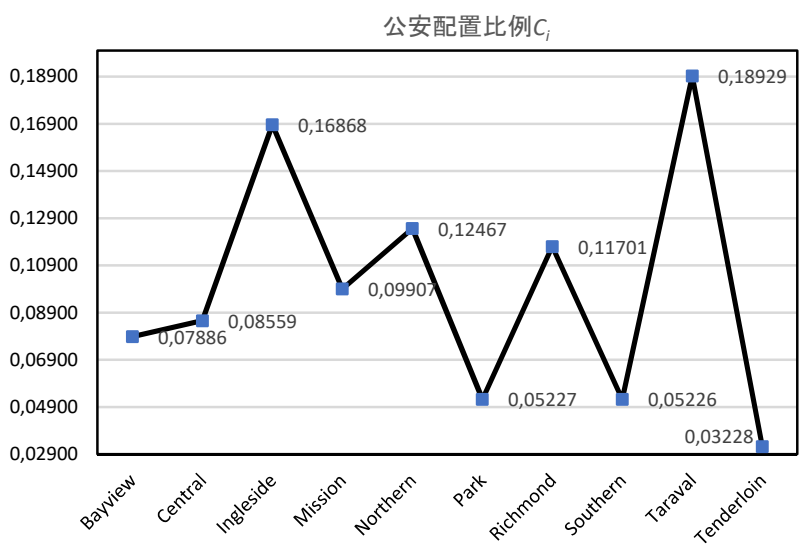

图 6.各区域公安配置比例

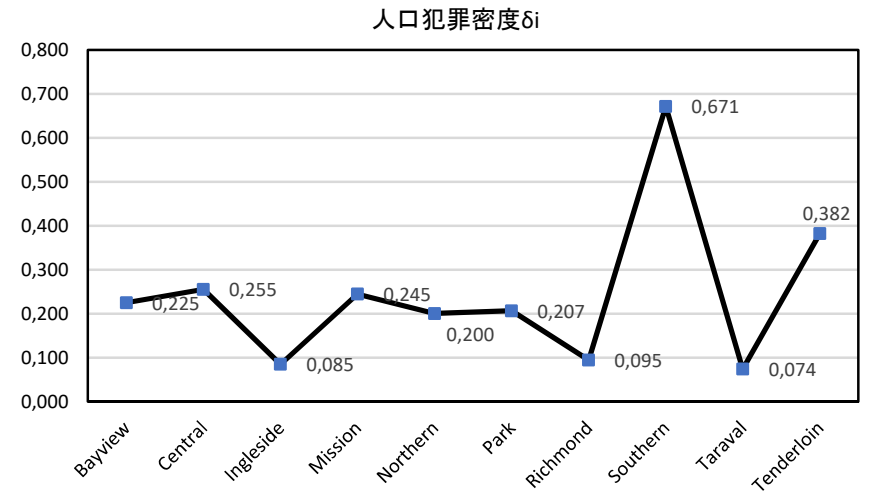

图 7.各区域人口犯罪密度

犯案成功率1- $\alpha_{i}$

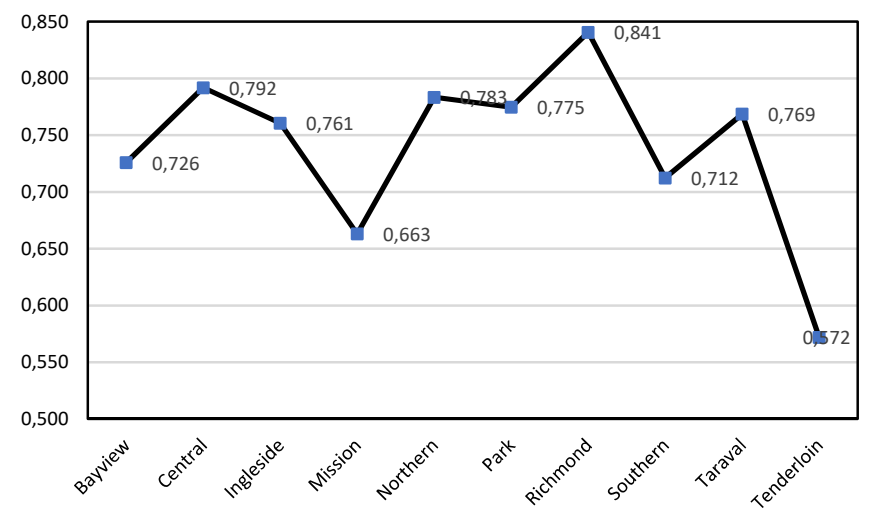

图 8. 各区域犯案成功率

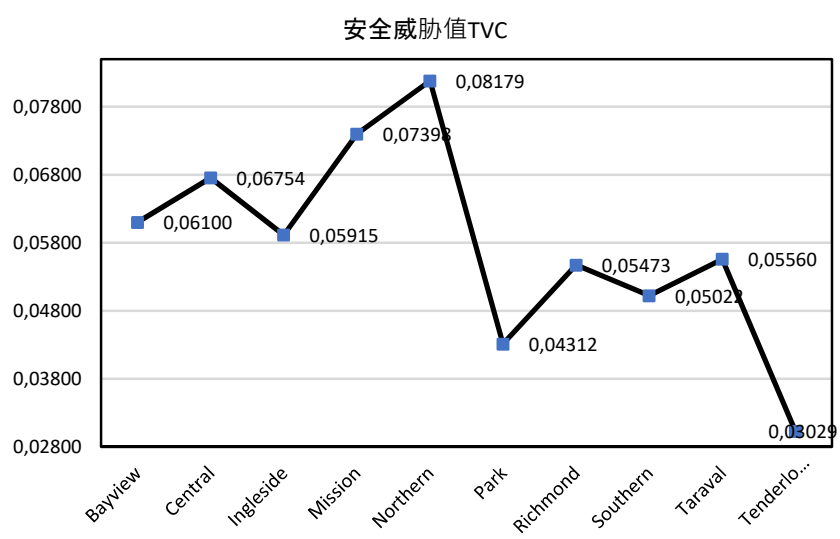

图 9. 各区域安全威胁值

按早、中、晚三时段「夏普利值」来分配公安警力: 本研究将早、中、晚三时段发生案件的次数的比例, 当 作三个安全警示等级的三个门坎值, 并分别计算三个时 段的各区域夏普利值, 如图 10。再将各区域的「夏普利 值」乘「各时段的公安数量」, 得到每日三时段的公安 巡逻配置图, 如图 11。在图 11中, 我们可以看到, 在安 全等级较高时, 即晚班时段, 城市里针对安全威胁值高 的 Northern、Mission、Central 等区域配置较高的公安巡 逻数量, 达到「抓大放小」的取舍。在安全等级较低时, 
即早班时段，管理者可以把所有的公安巡逻警力尽量均 分给每一区域, 达到「防微杜渐」的功能。在现有的公 安巡逻警力下，可以有效的分配整体三时段的巡逻班表。 公安部门管理者应用本架构可以量化评估那个区域是较 危险？以增加公安部门处理犯罪发生时的反应能力，并 可以让各区域公安警力依据本研究计算的配置表，互相 支持达到跨区查案的功能。

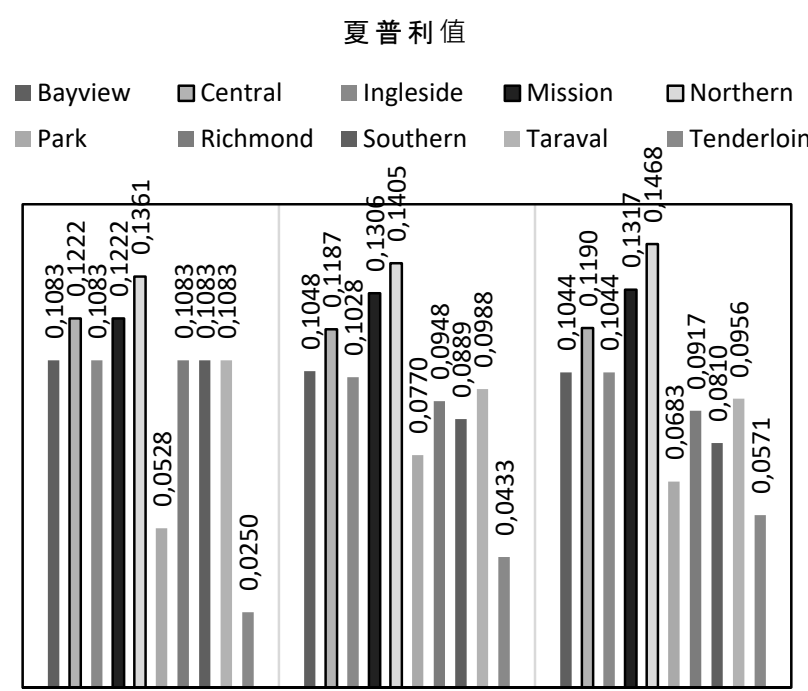

00:00 07:59 早 08:00 15:59 中 16:00 23:59 晚 班 班 班

图 10.各区域每日三时段的夏普利值

每日三時段的公安巡逻班表

\begin{tabular}{|c|c|c|c|c|}
\hline Bayview & $\square$ Central & Ingleside & aission & $\square$ Northern \\
\hline Park & Richmond & - Southern & Taraval & $\underset{\square}{\text { Tenderloin }}$ \\
\hline
\end{tabular}

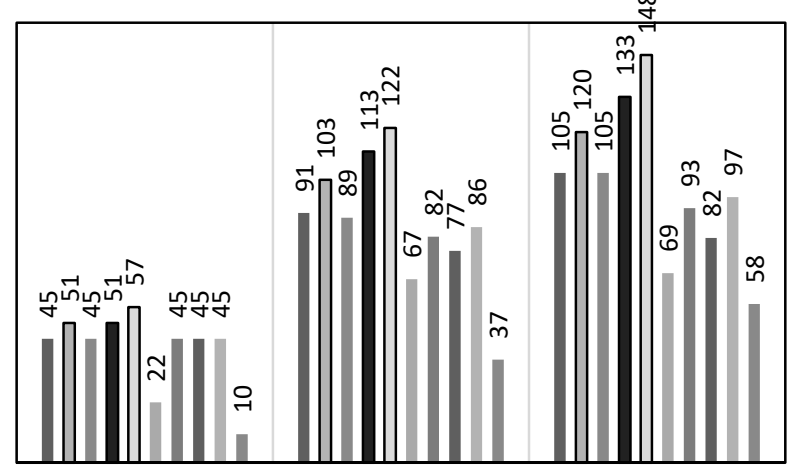

$00: 00 \sim 07: 59$ 早 $08: 00 \sim 15: 59$ 中 $16: 00 \sim 23: 59$ 晚 班 班 班

\section{IV. 结论与讨论}

本研究和一般计算安全威胁值不同, 主要是利用博亦 理论的方法, 来解析公安领导和犯罪者间的互动行为, 将两者的相互关联行为, 建立在一个非合作零和博弯。 我们在分析中可以看出, 如果单单只是从犯罪者的观点, 即人口数量、犯案成功率来检视区域的安全威胁值, 或 者以公安领导者的观点, 即人口犯案密度、公安配置比 例来检视区域的安全威胁值, 都不够周延, 惟有同时考 虑两者的观点, 相信必会得到公安警务人员的赞同。由 计算前各区域的公安配置数量, 按早、中、晚三时段的 夏普利值来重新分配公安巡逻警力。

本研究如果应用在城市公安治理, 得到良好的满意度, 可以计算每年巡逻警力配置表, 以这表作为公安警力人 员岗位数量的配置依据, 经由调整各区域公安警力到优 化的配置状态, 以提升公共整体安全。未来应用在公安 部门的排班系统, 预测会和实施的排班系统产生若干的 落差与不同, 需要与公安警务人员及系统管理人员沟通 协调，并做适当的调整。

\section{参考文献}

[1] C. Zhang, A. Sinha , and Tambe M. "Keeping pace with criminals: Designing patrol allocation against adaptive opportunistic criminals," pp. 1351-1359 in Proceedings of the 2015 international conference on Autonomous agents and multiagent systems, 2015.

[2] A. E. Roth, and E. V. Robert, "The Shapley Value as Applied to Cost Allocation: A Reinterpretation," Journal of Accounting Research, 17: pp.295-303, 1979.

[3] SF OpenData. SFPD Incidents - Year 2016. Available at: https://data.sfgov.org/Public-Safety/SFPD-Incidents-Previous-Year2016-/ritf-b9ki. Accessed 16 April 2017.

[4] C.E. Lemke, J.T Howson, "Equilibrium Points of Bimatrix Games." SIAM J. Applied Math. 12: 413-423, 1964.

[5] A, Dixit and S. Skeath Games of Strategy, W. W. Norton \& Company, 2007.

[6] M. J. Osborne and A. Rubinstein, A Course in Game Theory. MIT Press, 1994

[7] D. Mishra and B. Rangarajan, "Cost Sharing in a Job Scheduling Problem Using the Shapley Value", pp. 232-239 in Proceedings of the 6th ACM conference on Electronic commerce, 2005.

[8] J. M. Zolezzi, H. Rudnick. "Transmission cost allocation by cooperative games and coalition formation," IEEE Power Engineering Review, vol.17(4), pp.1008-1015, 2002.

图 11.各区域每日三时段的公安巡逻配置 